\title{
Advances of QIRT in medical diagnostics
}

by A. Nowakowski*

*Gdansk University of Technology - Department of Biomedical Engineering, Gdansk, Poland

\section{Abstract}

Recent advances of quantitative IR thermal imaging in medical diagnostics are discussed. The main interest is devoted to active dynamic thermography (ADT) in diagnostics of burn wounds and in monitoring of cardiosurgery interventions. Such applications must be taking into account basic problems existing in medical applications as: low thermal conductivity of biological tissues; influence of different metabolic processes on temperature distribution; need for elimination of registration errors caused by natural behaviour of a living patient, as breathing or accidental movements; finally a difficult problem of understanding conditions of energy exchange influencing heat flow in a living tissue/organ.

\section{Introduction}

IR-thermal imaging is present in medical applications since sixties of the $\mathrm{XX}$ century. After first fascination of this technology and applications to diagnostics of such diseases as breast tumour and in many other fields of medicine, after several years of using IR-thermal imaging in clinical practice a very negative opinion was established due to non proper use of instrumentation and lack of standard procedures, stopping for many years development of this technology in medicine. The recovery of IR-thermography in medical diagnostics is observed for more than ten years, now, due to recent developments of IR-imaging and also development of quantitative methods using digital signal processing. Very important was also development of QIRT methods in nondestructive material testing, giving an impulse to apply such methods in medicine, too. The state of the art in this field is given in the recently issued monograph [1]. Some of successfully developed and applied in medicine methods are here shortly presented. Our notice is focused on application of quantitative IR-thermal imaging and active dynamic thermography in monitoring of cardio surgical interventions and in diagnostics of skin burns to discriminate regions for surgical treatment.

\section{QIRT methods and Active Dynamic Thermography}

QIRT methods are using different mathematic formulas for evaluation of the content of thermal images. Some of the methods will be here characterised from the point of view of medical applications. Important progress comes due to applications of modern, high thermal resolution cameras and use of additional factors, as e.g. delicate cooling in breast cancer diagnostics. Our main area of interest is development of Active Dynamic Thermography (ADT) and procedures of Thermal Tomography (TT) in medicine [1]. This in fact is the use of the pulsed thermography and application of advanced data analysis in comparison with thermal models of tested objects/tissues. Main problems of practical development of ADT and TT are accuracy in analysis of series of thermal images, collected during or after external thermal excitation. In medical applications the analysis of heat flows is not as easy as in the case of material testing. We are dealing here with a living object so images are affected by complicated influence of motion, metabolic processes and some other factors. First, it is necessary to eliminate unwanted factors responsible for errors induced in sequences of images collected in time. Also the problem of heat exchange is complicated, as the surface temperature is usually lower than the internal body temperature, what is strongly affecting the way of heat exchange and may lead to erroneous conclusions.

\section{Monitoring in cardiosurgery}

The results of the research grant "Analysis of diagnostic and therapeutic procedures to minimise the risk of cardiosurgical interventions" performed during last three years will be here presented. Among several different diagnostic methods we have been studying we found that both, thermal imaging as well as ADT,are very useful for monitoring of surgical procedures quality of the heart during open chest operations. This problem is still very important as up to $5 \%$ of such interventions may be affected by more or less serious complications. Thermal imaging seems to be an almost ideal method, as it is fully aseptic, safe, not disturbing medical staff and performed on place and on-time. Basic results will be illustrated by examples of in vivo animal experiments as well as some clinical examples. As an example, in Fig.1 thermal images of the heart for in vivo animal experiments are shown. Fast decrease of temperature after stopping blood flow in the LAD is visible. 


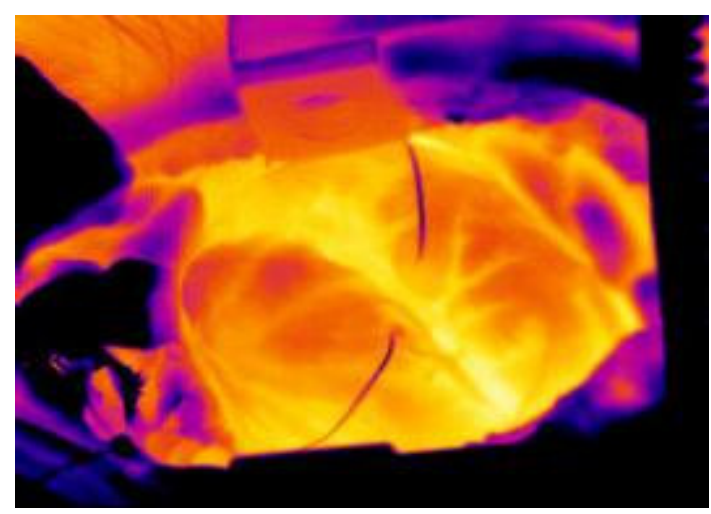

$0 \mathrm{~s}$
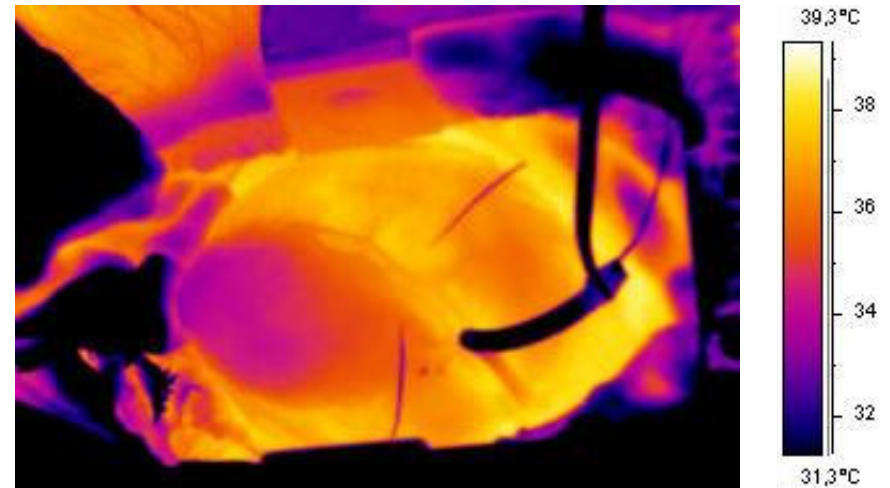

$60 \mathrm{~s}$

Fig.1. Thermal images of a pig heart at the beginning of clamping the left descending artery and after $60 \mathrm{~s}$.

\section{Diagnostics of skin burns}

Thermal imaging in diagnostics of burns is well known since early applications of this modality in medicine. Still the value of such diagnostics is limited. We applied procedures of ADT for such applications. It seems that now our proposal is competing with some other new methods, as laser Doppler imaging, for acceptance in clinical practice. Our experiments and theoretical studies prove that ADT is much more sensitive than simple thermography and may be directly applied for discrimination of burned regions requiring surgical treatment, what is still one of the main problems in burn diagnostics.

\section{REFERENCES}

[1] N. A. Diakides, J.D. Bronzino editors. Medical Infrared Imaging. CRC Press Taylor \& Francis Group, 2008. 